\title{
Synthesis of Coumarins and Quinolones by Intramolecular Aldol Condensation Reactions of Titanium Enediolates
}

\author{
Alois Fürstner*, Denis N. Jumbam, Nongyuan Shi \\ Max-Planck-Institut für Kohlenforschung, Kaiser-Wilhelm-Platz 1, \\ D-45470 Mülheim a.d. Ruhr \\ Dedicated to Prof. Dr. Dr. h.c. mult. Günther Wilke on the occasion of his 70th birthday \\ Z. Naturforsch. 50 b, 326-332 (1995); received September 13, 1994 \\ Low-Valent Titanium, Enediolates, Coumarins, 2-Quinolones, Aldol Condensation \\ Low-valent titanium prepared by the reduction of $\mathrm{TiCl}_{3}$ with zinc dust oxidatively adds to \\ $\alpha$-ketoamides or $\alpha$-ketoesters with the formation of the corresponding titanium enediolates. \\ These 1,2-difunctional nucleophiles, which have hardly been used in organic synthesis so far, \\ undergo regioselective intramolecular aldol condensation reactions with various electrophiles \\ such as aldehydes, ketones, nitriles, esters and amides. This methodology allows the synthesis \\ of differently substituted coumarin and 2-quinolone derivatives.
}

Low-valent titanium [Ti] prepared by the reduction of $\operatorname{TiCl}_{x}(x=3,4)$ with various reducing agents is a versatile tool for organic synthesis. Due to its high oxophilicity and electron transfer ability it promotes i.a. the reductive coupling of aldehydes or ketones to olefins, generally referred to as "McMurry reaction" [1]. A pronounced template effect of the titanium species makes the cyclization of dicarbonyl compounds particularly favourable and allows the formation of cycloalkenes independent of the ring size. These specific features are responsible for the widespread applications of this reaction in the synthesis of natural and non-natural products [1].

Its most important short-coming, however, stems from the limitation to aldehydes and ketones as essentially the only substrates that undergo such reductive $\mathrm{C}-\mathrm{C}$ bond formations [1]. Only recently we have been able to extend the scope of titanium-promoted processes beyond these traditional starting materials. Intramolecular alkylidenations of esters or amides as depicted in Scheme 1 give a ready access to the furan, pyrrole,

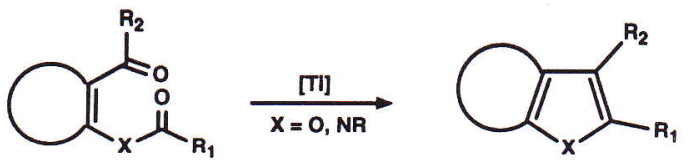

Scheme 1.

* Reprint requests to Priv.-Doz. Dr. A. Fürstner. benzo[b]furan and indole series [2-4]. In pursuing this concept, we now describe a complementary approach to six-membered heterocycles based upon the oxidative addition of low-valent titanium to 1,2-dicarbonyl compounds.

\section{Results and Discussion}

It is well established that activated titanium [Ti] readily deoxygenates acyloin derivatives most likely via the respective titanium enolates [2a, 5]. Although early attempts to trap these intermediates had failed [5], we were able to intercept one of them in an entropically favoured intramolecular way leading to the formation of the 4-quinolone 2 from substrate $\mathbb{1}$ (Scheme 2) [4].<smiles>COC(=O)c1ccccc1NC(=O)COc1cccc2[nH]c(-c3ccccc3)cc(=O)c12</smiles>

Scheme 2.

Although the reactivity of 1,2-dicarbonyl compounds might by closely tied to that of acyloins titanium-mediated transformations of this group of substrates have been even less studied. There is some precedence in the literature that they afford the respective enediolates upon treatment with low-valent metals (Scheme 3). As early as 1972, 
Floriani et al. reported a successful oxidative addition of $\mathrm{Cp}_{2} \mathrm{Ti}(\mathrm{CO})_{2}$ to 9,10-phenanthrenequinone [6]. Schobert et al. have later on extended this chemistry to other vicinal diketones and derivatives thereof using "reactive titanocene" (formed by reduction of $\mathrm{Cp}_{2} \mathrm{TiCl}_{2}$ with $\mathrm{Mg}$ ) as the reagent. The 1,2-difunctional nucleophiles thus obtained were trapped with strong electrophiles such as $\mathrm{H}^{+}$, phosgene, $\mathrm{PhBCl}_{2}, \mathrm{PhPCl}_{2}$ and acid chlorides [7]. Similarly, $\alpha$-ketoesters have been used in lowvalent titanium-mediated aldol reactions [8].

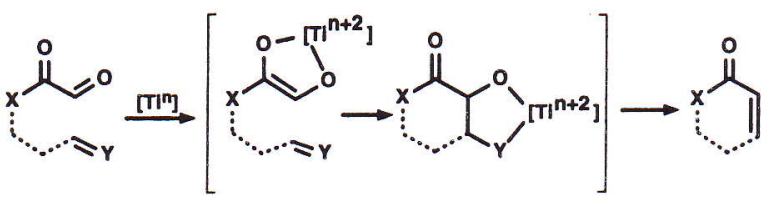

Scheme 3.

We intended to devise intramolecular versions of such reactions (Scheme 3). Being entropically biased, they might allow to intercept the titanium enediolates with less reactive electrophilic groups such as aldehydes, ketones, esters, amides or nitriles.

A set of suitable substrates $4 \mathbf{a - k}$ and $6 \mathbf{6 a}, \mathbf{b}$ was prepared by the acylation of commercially available compounds $\mathbf{3}$ and $\mathbf{5}$ with different $\alpha$-ketoacid chlorides [9] under standard conditions (Scheme 4, Table II). In case of $X=O$ only moderate yields have been obtained because of the low nucleophilicity of the phenolic $-\mathrm{OH}$ and of partial hydrolysis of the esters $4 \mathbf{i}, \mathbf{j}$ and $6 \mathbf{a}$ during flash chromatography.

In a previous study [3] we reported the cyclization of substrate $4 \mathrm{a}$ to the simple indole alkaloid salvadoricine 7 in $60 \%$ yield as the only product that could be isolated from the crude reaction mixture (Scheme 5). The striking chemoselectivity of this transformation in favour of the ketone-amide rather than the conventional ketone-ketone coupling process together with the complete resistance of the carbonyl group of $\mathbf{7}$ to an excess of the titanium reagent used are without precedence in the literature.

This result, however, turned out to be an exception to the rule, since all other substrates of this series followed a different pathway as can be seen from the data compiled in Table I. Thus, compounds $4 \mathbf{b}-\mathbf{f}$ readily cyclized to the corresponding

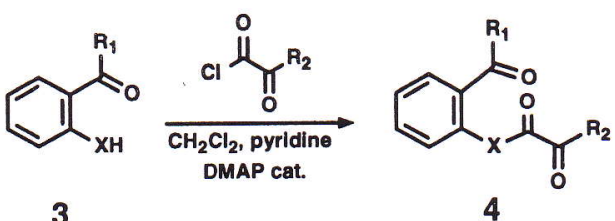

3

\begin{tabular}{|c|c|c|c|}
\hline & $x$ & $R_{1}$ & $\mathbf{R}_{2}$ \\
\hline & NH & Me & Me \\
\hline & NH & $\mathbf{H}$ & Ph \\
\hline & $\mathrm{NH}$ & Me & $\begin{array}{l}\mathrm{Ph} \\
\mathrm{Ph}\end{array}$ \\
\hline & NH & $\mathrm{Ph}$ & Me \\
\hline & NH & Me & 2-Thienyl \\
\hline & NH & OMe & $\mathrm{Ph}$ \\
\hline & $\underset{0}{\text { NH }}$ & $\begin{array}{l}\mathrm{NH}_{2} \\
\mathrm{Me}^{2}\end{array}$ & $\begin{array}{ll}P h \\
M B\end{array}$ \\
\hline & o & Me & $\mathrm{Ph}$ \\
\hline & 0 & OMe & $\mathrm{Ph}$ \\
\hline
\end{tabular}

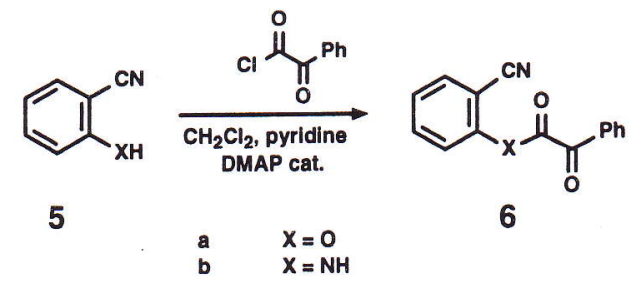

Scheme 4

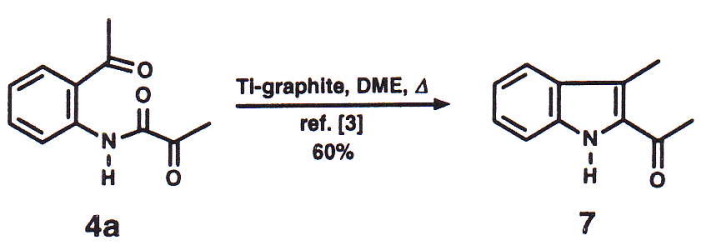

Scheme 5 .

2-quinolone derivatives $\mathbf{8 b - \mathbf { f }}$ in good yields (Scheme 6). Indoles were not observed, except in the case of substrate $4 \mathbf{c}$ where a minute amount of 2-benzoyl-3-methylindole $(\approx 5 \%)$ accompanied the formation of quinolone $8 \mathrm{c}$. Similarly, esters $4 \mathbf{i}, \mathbf{j}$ afforded the corresponding 3,4-disubstituted coumarins $8 \mathbf{i}$, $\mathbf{j}$ without incident (Scheme 6).

Entries 6, 7, 10-13 in Table I comprise those cases in which we tried to intercept the intermediate enediolates with electrophilic groups other than an aldehyde or a ketone. An adjacent ester or a carboxylic acid amide group were only partly suitable for this purpose. Compound $\mathbf{4} \mathbf{g}$ gave a 
<smiles>[Y][Y](=O)C(C)=O</smiles>

4<smiles>COC(=O)c1ccccc1NC(=O)C(=O)Nc1ccccc1-c1ccccc1</smiles><smiles>Cc1ccccc1I=CC(=O)C(=O)c1ccccc1</smiles>

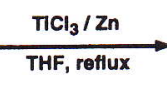

6

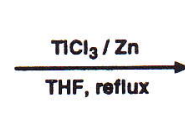<smiles>[R]c1c(Br)c2ccccc2[Y]c1=O</smiles>

8
Scheme 6.

Table I. Low-valent titanium-induced 2-quinolone and coumarin syntheses. Method $\mathbf{A}: \mathrm{TiCl}_{3}$ was reduced with $\mathrm{Zn}$-dust prior to the addition of the substrate [14]; Method B: reduction of $\mathrm{TiCl}_{3}$ with $\mathrm{Zn}$-dust in the presence of the substrate [4] ( $c f$. Experimental section).

\begin{tabular}{rllcr}
\hline Entry & Substrate & Method & $\begin{array}{c}\text { Reaction- } \\
\text { time }[\mathrm{h}]\end{array}$ & $\begin{array}{l}\text { Product } \\
\text { [yield \%] }\end{array}$ \\
\hline 1 & $\mathbf{4 b}$ & $\mathrm{A}$ & 5 & $\mathbf{8 b}(64 \%)$ \\
2 & $\mathbf{4 c}$ & $\mathrm{A}$ & 1 & $\mathbf{8 c}(80 \%)^{\mathrm{a}}$ \\
3 & $\mathbf{4 d}$ & $\mathrm{A}$ & 16 & $\mathbf{8 d}(68 \%)$ \\
4 & $\mathbf{4 e}$ & $\mathrm{A}$ & 16 & $\mathbf{8 e}(69 \%)$ \\
5 & $\mathbf{4 f}$ & $\mathrm{A}$ & 16 & $\mathbf{8 f}(50 \%)$ \\
6 & $\mathbf{4} \mathbf{g}$ & $\mathrm{B}$ & 3 & $\mathbf{1 0}(41 \%)$ \\
& & & & $\mathbf{1 1}(15 \%)$ \\
7 & $\mathbf{4 h}$ & $\mathrm{A}$ & 16 & $\mathbf{1 2 b}(40 \%)$ \\
8 & $\mathbf{4 i}$ & $\mathrm{A}$ & 0.5 & $\mathbf{8 i}(80 \%)$ \\
9 & $\mathbf{4 j}$ & $\mathrm{A}$ & 1 & $\mathbf{8 j}(76 \%)$ \\
10 & $\mathbf{4 k}$ & $\mathrm{A}$ & 16 & $\mathbf{3 k}(79 \%)$ \\
11 & $\mathbf{6 a}$ & $\mathrm{A}$ & 1 & $\mathbf{1 2 a}(85 \%)$ \\
12 & $\mathbf{6 a}$ & $\mathrm{B}$ & 4 & $\mathbf{1 2 a}(86 \%)$ \\
13 & $\mathbf{6 b}$ & $\mathrm{A}$ & 5 & $\mathbf{1 2 b}(45 \%)$ \\
\hline
\end{tabular}

a Together with 2-benzoyl-3-methylindole $(\approx 5 \%)$.

mixture of the desired 4-hydroxy-2-quinolone $\mathbf{1 0}$ together with substantial amounts of the uncyclized deoxygenation product 11 [10]. Under the same conditions the phenylglyoxylate $4 \mathbf{k}$ was cleaved to the parent methyl 2-hydroxybenzoate 3k. A nitrile group, however, turned out to be suitable to trap the titanium enediolates, since compounds $6 \mathbf{a}, \mathbf{b}$ gave the 4 -amino-substituted deriva- tives $\mathbf{1 2} \mathbf{a}, \mathbf{b}$ in reasonable to good yields (Table I, entries 11-13).

Remarkably, the results obtained proved to be highly dependent on the type of low-valent titanium [Ti] used. While in the salvadoricine synthesis titanium-graphite prepared by the reduction of $\mathrm{TiCl}_{3}$ with potassium-graphite laminate $\left(\mathrm{C}_{8} \mathrm{~K}\right)$ [11] turned out to be suited best, this reagent failed to cyclize substrate $4 \mathrm{c}$ but led to the simple reduction of the $\alpha$-ketoamide group (Scheme 7). The formation of compound 9 may be explained via the protonation of an intermediate titanium enediolate during work-up. Ammonium ion stabilized titanium clusters obtained from $\mathrm{TiCl}_{4} /$ $\mathrm{K}\left(\mathrm{BEt}_{3} \mathrm{H}\right)$ [12] gave the same product. $\mathrm{TiCl}_{3}$ itself, which has previously been employed to mediate pinacol cross-coupling reactions of methyl phenylglyoxylate with aldehydes and ketones [13], also afforded 9 in comparable yield. In contrast, the titanium species prepared from $\mathrm{TiCl}_{3}$ and zinc dust either prior to the addition of the substrate (method A) [14] or in its presence (method B) [4] promoted the desired condensation reactions and have therefore been used throughout this study.

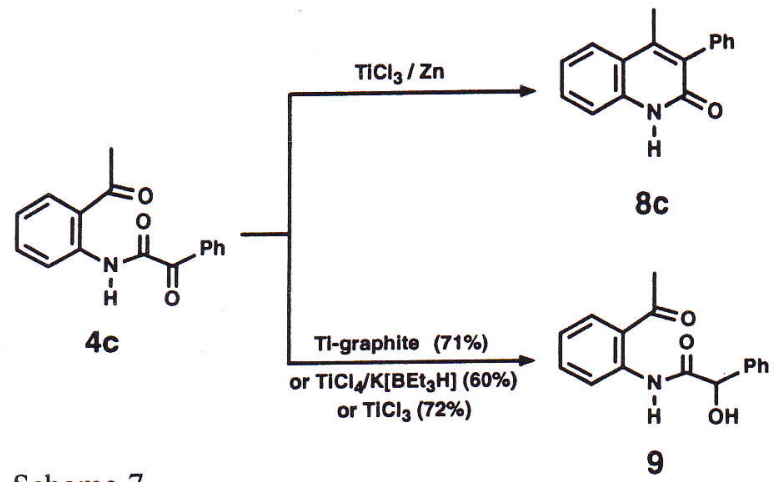

Scheme 7.

These results highlight the empirical state of the art of low-valent titanium chemistry, where subtle changes in the reagent preparation may have great impact for the outcome of the reaction [1]. In view of the lack of reliable information on the actual nature of such "low-valent" titanium species an accurate interpretation of these results on a morphological basis is presently impossible [15].

Applications of these titanium-mediated reactions to the synthesis of heterocyclic natural products and of pharmacologically active compounds are in progress. 


\section{Experimental}

All reactions were carried out in pre-dried glassware under argon using Schlenk techniques. Melting points were recorded on a Gallenkamp apparatus and are uncorrected. NMR: Bruker AM 200 at $200 \mathrm{MHz}\left({ }^{1} \mathrm{H}\right)$ and $50 \mathrm{MHz}\left({ }^{13} \mathrm{C}\right)$, respectively, in $\mathrm{CDCl}_{3}$ with TMS as internal standard unless stated otherwise. MS: EI, Finnigan MAT $311 \mathrm{~A}$ $(70 \mathrm{eV})$. IR: Nicolet FT-510. TLC: Polygram ${ }^{\circledR}$ Sil $\mathrm{Gel} \mathrm{UV}_{254}$ (Macherey, Nagel \& Co.). Flash chromatography: Merck silica gel 60 (230-400 mesh) with hexane/ethyl acetate in various proportions as eluent. $\mathrm{TiCl}_{3}$ : Aldrich, 99\%. Anhydrous solvents were obtained by distillation over the given drying agents: THF (potassium/benzophenone), DME (Na/K alloy), $\mathrm{CH}_{2} \mathrm{Cl}_{2}\left(\mathrm{CaH}_{2}\right)$, pyridine (MS $4 \AA$ ). The 2-ketoacids were purchased from Aldrich and used without further purification. The corresponding acid chlorides have been prepared according to the literature [9].

\section{Representative procedure for acylation reactions with 2-ketoacid chlorides}

Phenylglyoxylic acid chloride $(0.80 \mathrm{~g}, 4.74 \mathrm{mmol})$ [9] in $\mathrm{CH}_{2} \mathrm{Cl}_{2}(5 \mathrm{ml})$ was added dropwise to a solution of 2-hydroxybenzonitrile $(0.50 \mathrm{~g}, 4.20 \mathrm{mmol})$ and a catalytic amount of 4-dimethylaminopyridine (ca. $50 \mathrm{mg}$ ) in $\mathrm{CH}_{2} \mathrm{Cl}_{2}(25 \mathrm{ml})$. The mixture was stirred at ambient temperature for $4 \mathrm{~h}$, quenched with a saturated aqueous solution of $\mathrm{NaHCO}_{3}$, the aqueous layer was extracted twice with $\mathrm{CH}_{2} \mathrm{Cl}_{2}(50 \mathrm{ml}$ each $)$, the combined organic phases were dried over $\mathrm{Na}_{2} \mathrm{SO}_{4}$, the solvent was evaporated and the residue was purified by flash chromatography with hexane/ethyl acetate (gradient $10 / 1 \rightarrow 4 / 1$ ) as eluent. All other $\alpha$-ketoesters and $\alpha$-ketoamides were prepared analogously. For yields, analytical and spectroscopic data see Table II.

\section{General procedure for the formation of 2-quinolones and coumarins}

Method A: A suspension of $\mathrm{TiCl}_{3}(771 \mathrm{mg}$, $5.0 \mathrm{mmol}$ ) and zinc dust $(653 \mathrm{mg}, 10.0 \mathrm{mmol})$ in anhydrous THF $(50 \mathrm{ml})$ was refluxed under Ar for $4 \mathrm{~h}$. After a solution of the respective substrate $(2.5 \mathrm{mmol})$ in THF $(10 \mathrm{ml})$ was added rapidly to the boiling black mixture of the active titanium reagent, reflux was continued until TLC showed the complete conversion of the starting material. The reaction mixture was then allowed to cool to ambient temperature, filtered through a short pad of silica, the insoluble residue was washed with
THF $(\approx 30 \mathrm{ml})$ and ethyl acetate $(\approx 50 \mathrm{ml})$ in several portions, the combined filtrates were evaporated and the residue chromatographed with hexane/ethyl acetate in various proportions. For the yields obtained and the reaction times see Table I. The analytical and spectroscopic data of the products ( $c f$. Table I) are compiled below.

\section{3-Phenyl-2-quinolone $(\mathbf{8 b})$}

M.p. $198-200^{\circ} \mathrm{C}$ (dec.) (lit. $235^{\circ} \mathrm{C}$ [16]). - IR $\left(\mathrm{cm}^{-1}\right): 2850-3250,1660,1570,1495,750,700$. ${ }^{1} \mathrm{H}$ NMR (DMSO-d ${ }_{6}$ ): $\delta=11.94$ (br s, $1 \mathrm{H}$ ), 8.11 (s, 1H), 7.71-7.76 (m, 3H), 7.32-7.51 (m, 5H), $7.18(\mathrm{dt}, J=0.6,3.8,1 \mathrm{H}) .-{ }^{13} \mathrm{C}$ NMR (DMSO$\left.\mathrm{d}_{6}\right): \delta=160.7,138.0,137.3,135.9,131.2,129.8$, $128.3,127.8,127.6,127.4,121.6,119.2,114.3$. - MS: $\mathrm{m} / \mathrm{z}$ (rel. intensity, \%): $221\left(\left[\mathrm{M}^{+}\right], 100\right), 193(7)$, 165 (15), 110 (8), 89 (8).

\section{4-Methyl-3-phenyl-2-quinolone (8c)}

M.p. $262-264{ }^{\circ} \mathrm{C}$. - IR $\left(\mathrm{cm}^{-1}\right): 2750-3100$, $1650,1610,1600,1560,1500,1430,1380,1285,980$, $890,750,705 .-{ }^{1} \mathrm{H}$ NMR (DMSO-d 6 ): $\delta=12.03$ (br s, $1 \mathrm{H}), 7.86(\mathrm{~d}, J=8.5,1 \mathrm{H}), 7.18-7.56(\mathrm{~m}$, $8 \mathrm{H}), 3.35(\mathrm{~s}, 3 \mathrm{H}) .-{ }^{13} \mathrm{C}$ NMR $\left(\mathrm{DMSO}_{-} \mathrm{d}_{6}\right): \delta=$ 161.0, 143.2, 137.8, 136.3, 132.0, 130.3, 130.0, 127.8, $127.1,125.2,121.7,119.9,115.1,16.5$. - MS: $\mathrm{m} / \mathrm{z}$ (rel. intensity, \%): 235 ([M $\left.\left.{ }^{+}\right], 67\right), 234$ (100), 216 (14).

\section{$N$-(2'-Acetylphenyl)-2-hydroxy-phenylacetamide} (9)

IR $\left(\mathrm{cm}^{-1}\right):$ 2900-3500, 1655, 1580, 1530, 1455, 1250, 750. - ${ }^{1} \mathrm{H}$ NMR (DMSO-d 6 ): $\delta=12.43$ (br s, $1 \mathrm{H}), 8.60(\mathrm{~d}, J=8.4,1 \mathrm{H}), 8.09(\mathrm{dd}, J=1.5,8$, $1 \mathrm{H}), 7.18-7.63(\mathrm{~m}, 7 \mathrm{H}), 6.81(\mathrm{~d}, J=4), 5.10$ (d, $J=4,1 \mathrm{H}), 3.39(\mathrm{~s}, 3 \mathrm{H}) .-{ }^{13} \mathrm{C}$ NMR (DMSO-d 6 ): $\delta=202.2,172.1,140.3,138.9,134.3,132.1,128.0$, $127.5,126.3,122.7,119.4,73.9,28.6$. - MS: $m / z$ (rel. intensity, \%): $269\left(\left[\mathrm{M}^{+}\right], 10\right), 251$ (49), $222(9)$, 146 (34), 136 (100), 120 (54), 105 (17), 92 (16), 77 (22).

\section{3,4-Diphenyl-2-quinolone (8d)}

M.p. $303-305^{\circ} \mathrm{C}$ (lit. $305-307^{\circ} \mathrm{C}, 309-310^{\circ} \mathrm{C}$ $(\mathrm{EtOH})$ [17]). - IR $\left(\mathrm{cm}^{-1}\right): 2700-3200,1645$, 1595, 1570, 1500, 1485, 1440, 1430, 1375, 1290, 760, 700, 670, 650, 610, 560. - ${ }^{1} \mathrm{H}$ NMR (DMSO-d ${ }_{6}$ ): $\delta=12.10$ (br s, $1 \mathrm{H}), 6.97-7.53(14 \mathrm{H}) .-{ }^{13} \mathrm{C} \mathrm{NMR}$ $\left(\right.$ DMSO- $\left.\mathrm{d}_{6}\right): \delta=160.9,147.8,138.0,135.8,135.4$, $131.7,130.4,129.8,129.2,127.7,127.2,126.8,126.5$, 126.3, 121.4, 119.6, 114.9. - MS: $m / z$ (rel. intensity, \%): 297 ([M+], 59), 296 (100), 278 (17), 267 (10). 
Table II. Analytical and characteristic spectroscopic data of the starting materials.

\begin{tabular}{|c|c|c|c|c|c|c|}
\hline $\begin{array}{l}\text { Com- } \\
\text { pound }\end{array}$ & $\begin{array}{l}\text { Yield } \\
{[\%]}\end{array}$ & $\begin{array}{l}\mathrm{m} . \mathrm{p} . \\
{\left[{ }^{\circ} \mathrm{C}\right]}\end{array}$ & $\operatorname{IR}\left[\mathrm{cm}^{-1}\right]$ & MS: $m / z[\%]$ & ${ }^{1} \mathrm{H}$ NMR $[\delta]$ & ${ }^{13} \mathrm{C}$ NMR $[\delta]$ \\
\hline $4 b$ & 71 & $120-122$ & $\begin{array}{l}3240,1700 \\
1680,1665\end{array}$ & $\begin{array}{l}253\left(\left[\mathrm{M}^{+}\right], 12\right), \\
105(100)\end{array}$ & $\begin{array}{l}12.46(\mathrm{br} \mathrm{s}, 1 \mathrm{H}, \mathrm{NH}) \\
10.01(\mathrm{~s}, 1 \mathrm{H}, \mathrm{CHO})\end{array}$ & $195.1,186.9,160.9$ \\
\hline $4 c$ & 88 & $107-108$ & $3190,1685,1645$ & $\begin{array}{l}267\left(\left[\mathrm{M}^{+}\right], 6\right) \\
162(100)\end{array}$ & $\begin{array}{l}12.93(\text { br s, } 1 \mathrm{H}, \mathrm{NH}) \text {, } \\
2.67(\mathrm{~s}, 3 \mathrm{H},-\mathrm{Me})\end{array}$ & $\begin{array}{l}201.8,186.7,160.3 \text {, } \\
28.0\end{array}$ \\
\hline $4 d$ & 80 & $132.5-133.5$ & $\begin{array}{l}3270,1690 \\
1670,1640\end{array}$ & $\begin{array}{l}329\left(\left[\mathrm{M}^{+}\right], 2\right), \\
224(100)\end{array}$ & 12.11 (br s, $1 \mathrm{H}, \mathrm{NH})$ & $198.4,186.6,159.9$ \\
\hline $4 e$ & 74 & $99-100$ & $3240,1690,1630$ & $\begin{array}{l}267\left(\left[\mathrm{M}^{+}\right], 5\right), \\
224(100)\end{array}$ & $\begin{array}{l}11.90(\text { br s, } 1 \mathrm{H}, \mathrm{NH}) \\
2.55(\mathrm{~s}, 3 \mathrm{H},-\mathrm{Me})\end{array}$ & $\begin{array}{l}\text { 198.6, 196.2, 158.7, } \\
24.0\end{array}$ \\
\hline $4 f$ & 75 & $149-150$ & $3150,1695,1655$ & $\begin{array}{l}273\left(\left[\mathrm{M}^{+}\right], 7\right), \\
162(100)\end{array}$ & $\begin{array}{l}13.07(\mathrm{br} \mathrm{s}, 1 \mathrm{H}, \mathrm{NH}), \\
2.68(\mathrm{~s}, 3 \mathrm{H},-\mathrm{Me})\end{array}$ & $\begin{array}{l}201.6,177.6,159.4 \text {, } \\
28.0\end{array}$ \\
\hline $4 g$ & 89 & $101-102$ & $3440,1710,1680$ & $\begin{array}{l}283\left(\left[\mathrm{M}^{+}\right], 7\right), \\
146(100)\end{array}$ & $\begin{array}{l}12.44(\mathrm{br} \mathrm{s}, 1 \mathrm{H}, \mathrm{NH}) \\
3.92(\mathrm{~s}, 3 \mathrm{H},-\mathrm{OMe})\end{array}$ & $\begin{array}{l}186.8,167.8,159.8, \\
52.4\end{array}$ \\
\hline $4 h$ & 76 & $181-184$ & $\begin{array}{l}3400,3190,1690 \\
1660,1610\end{array}$ & $\begin{array}{l}268\left(\left[\mathrm{M}^{+}\right], 1\right), \\
163(100)\end{array}$ & $\begin{array}{l}12.88\left(\mathrm{br} \mathrm{s}, 1 \mathrm{H}, \mathrm{NH}^{2}\right) \\
8.34\left(\mathrm{br} \mathrm{s}, 2 \mathrm{H}, \mathrm{NH}_{2}\right)^{\mathrm{c}}\end{array}$ & $187.6,170.1,160.1^{\mathrm{c}}$ \\
\hline $4 i$ & 40 & syrup & $1770,1740,1685$ & $\begin{array}{l}206\left(\left[\mathrm{M}^{+}\right], 1\right), \\
121(100)\end{array}$ & $\begin{array}{l}2.60,2.53 \\
(\mathrm{~s} \text { each, } 3 \mathrm{H},-\mathrm{Me})\end{array}$ & $\begin{array}{l}\text { 196.5, 190.5, 158.6, } \\
28.5,26.2\end{array}$ \\
\hline $4 j$ & 59 & $68-71$ & 1750,1690 & $105(100)$ & $2.58(\mathrm{~s}, 3 \mathrm{H},-\mathrm{Me})$ & $\begin{array}{l}196.8,184.3,160.8, \\
28.9\end{array}$ \\
\hline $4 k$ & 89 & $59-61$ & $1760,1715,1695$ & $105(100)^{\mathbf{a}}$ & $3.83(\mathrm{~s}, 3 \mathrm{H},-\mathrm{OMe})$ & $\begin{array}{l}184.6,164.6,161.3 \text {, } \\
52.3\end{array}$ \\
\hline $6 a$ & 58 & syrup & $2240,1760,1690$ & $105(100)^{b}$ & $\begin{array}{l}8.12-8.18(\mathrm{~m}, 2 \mathrm{H}), \\
7.36-7.75(\mathrm{~m}, 7 \mathrm{H})\end{array}$ & $\begin{array}{l}\text { 183.1, 159.6, 150.3, } \\
114.0,106.1\end{array}$ \\
\hline $6 \mathrm{~b}$ & 81 & $106-108$ & $\begin{array}{l}3330,2220 \\
1705,1665\end{array}$ & $\begin{array}{l}250\left(\left[\mathrm{M}^{+}\right], 9\right), \\
105(100)\end{array}$ & $9.51(\mathrm{br} \mathrm{s}, 1 \mathrm{H},-\mathrm{NH})$ & $\begin{array}{l}\text { 185.4, 158.6, 115.3, } \\
103.0\end{array}$ \\
\hline
\end{tabular}

${ }^{\text {a }}$ Chemical ionization $\left(\mathrm{NH}_{3}\right): 302\left(\left[\mathrm{M}+\mathrm{NH}_{4}\right]^{+}, 100 \%\right) ;{ }^{\mathrm{b}}$ chemical ionization $\left(\mathrm{NH}_{3}\right): 269\left(\left[\mathrm{M}+\mathrm{NH}_{4}\right]^{+}, 100 \%\right.$; ${ }^{\mathrm{c}}$ spectrum recorded in DMSO- $\mathrm{d}_{6}$.

\section{3-Methyl-4-phenyl-2-quinolone $(\mathbf{8} \mathbf{e})$}

M.p. $238-239^{\circ} \mathrm{C}$ (lit. $238-239{ }^{\circ} \mathrm{C}$ [18]). - IR $\left(\mathrm{cm}^{-1}\right): 2800-3190,1650,1610,1595,1560,1430$ 1370, 1280, 1020, 755, 705, 670, 610. $-{ }^{1} \mathrm{H}$ NMR $\left(\mathrm{DMSO}_{\mathrm{d}} \mathrm{d}_{6}\right): \delta=11.91$ (br s, $\left.1 \mathrm{H}\right), 7.20-7.58(\mathrm{~m}$, $7 \mathrm{H}), 7.01$ and $6.85(\mathrm{ddAB}, J=2,6.6,8.4,2 \mathrm{H}), 3.35$ (s, 3H). $-{ }^{13} \mathrm{C}$ NMR (DMSO-d $\mathrm{d}_{6}$ ): $\delta=161.7,146.7$, $137.1,136.3,128.9,128.5,128.4,128.0,127.7,126.8$, $125.8,121.2,119.7,114.8,13.9$. - MS: $m / z$ (rel. intensity, \%): $235\left(\left[\mathrm{M}^{+}\right], 56\right), 234$ (100), 216 (16).

\section{4-Methyl-3-(2'-thienyl)-2-quinolone (8f)}

M.p. $263-265^{\circ} \mathrm{C}$ - IR $\left(\mathrm{cm}^{-1}\right)$ : $2650-3180$, $1640,1600,1555,1505,1435,1385,1280,1235$, $1160,955,940,900,850,790,780,750,735,690$, $660,570 .-{ }^{1} \mathrm{H}$ NMR (DMSO-d 6 ): $\delta=11.87$ (br s, $1 \mathrm{H}), 7.79(\mathrm{dd}, J=0.6,4.2,1 \mathrm{H}), 7.65(\mathrm{dd}, J=0.6$, $2.4,1 \mathrm{H}), 7.51(\mathrm{dt}, J=0.6,3.8,1 \mathrm{H}), 7.33(\mathrm{dd}, J=$ $0.4,4.2,1 \mathrm{H}), 7.22(\mathrm{dt}, J=0.6,3.8,1 \mathrm{H}), 7.09-7.14$ $(\mathrm{m}, 2 \mathrm{H}), 3.35(\mathrm{~s}, 3 \mathrm{H}) .-{ }^{13} \mathrm{C}$ NMR (DMSO-d 6 ): $\delta=159.8,144.4,136.9,135.4,129.7,128.5,126.5$, 125.5, 124.9, 124.1, 121.3, 119.1, 114.5, 16.4. - MS: $\mathrm{m} / \mathrm{z}$ (rel. intensity, \%): $241\left(\left[\mathrm{M}^{+}\right], 100\right), 240(53)$, 208 (14), 196 (7).

\section{4-Hydroxy-3-phenyl-2-quinolone (10)}

M.p. $318-320^{\circ} \mathrm{C}$ (dec.) (lit. $\left.320^{\circ} \mathrm{C}[20]\right)$. - IR $\left(\mathrm{cm}^{-1}\right):$ 2700-3500, 1655, 1610, 1590, 1500, 1400, $1290,1160,1140,760,700,555 .-{ }^{1} \mathrm{H}$ NMR (DMSO-d 6 ): $\delta=11.47$ (br s, $1 \mathrm{H}$ ), 10.05 (br s, $1 \mathrm{H}$ ), $7.91(\mathrm{dd}, J=1.2,8,1 \mathrm{H}), 7.49(\mathrm{dt}, J=1.0,7,1 \mathrm{H})$, $7.24-7.42(\mathrm{~m}, 6 \mathrm{H}), 7.16(\mathrm{dt}, J=1.2,7,1 \mathrm{H}) .-{ }^{13} \mathrm{C}$ NMR (DMSO-d $\left.{ }_{6}\right): \delta=162.4,157.0,137.7,133.0$, $130.9,130.3,127.4,126.6,122.8,120.8,115.2$, 114.6, 112.4. - MS: $m / z$ (rel. intensity, \%): $237\left(\left[\mathrm{M}^{+}\right]\right.$, 100), 120 (87), 92 (25).

\section{$N$-(2'-Methoxycarbonylphenyl)-phenylacetamide (11)}

Syrup. - IR $\left(\mathrm{cm}^{-1}\right): 3300,3040,2960,1710$, $1690,1610,1590,1530,1450,1440,1300,1260$, 1090. - ${ }^{1} \mathrm{H}$ NMR: $\delta=11.05$ (br s, $\left.1 \mathrm{H}\right), 8.70$ (dd, $J=1,8.4,1 \mathrm{H}), 7.97(\mathrm{dd}, J=1.8,7.8,1 \mathrm{H}), 7.51(\mathrm{dt}$, $J=1.6,7.4,1 \mathrm{H}), 7.20-7.40(\mathrm{~m}, 5 \mathrm{H}), 7.05(\mathrm{dt}, J=$ $1.4,7.4,1 \mathrm{H}), 3.88(\mathrm{~s}, 3 \mathrm{H}), 3.75(\mathrm{~s}, 2 \mathrm{H}) .-{ }^{13} \mathrm{C}$ NMR: $\delta=169.6,168.0,140.9,134.0,130.3,129.1$, $128.4,126.8,122.1,120.0,114.8,107.4,51.8,45.5$. MS: $m / z$ (rel. intensity, \%): $269\left(\left[\mathrm{M}^{+}\right], 25\right), 178$ (44), 151 (49), 146 (100), 119 (33), 91 (38), 65 (13). 


\section{4-Amino-3-phenyl-2-quinolone (12b)}

M.p. $326-328^{\circ} \mathrm{C}$ (dec.) (lit. $\left.325^{\circ} \mathrm{C}[19]\right) .-$ IR $\left(\mathrm{cm}^{-1}\right): 3470,3320,2800-3250,1630,1610,1595$, $1510,1415,755,700 .-{ }^{1} \mathrm{H}$ NMR (DMSO) $\left.-\mathrm{d}_{6}\right): \delta=$ $11.04(\mathrm{~s}, 1 \mathrm{H}), 8.00(\mathrm{~d}, J=4,1 \mathrm{H}), 7.24-7.46(\mathrm{~m}$, $7 \mathrm{H}), 7.10(\mathrm{t}, J=3.6,1 \mathrm{H}), 5.86($ br s, $2 \mathrm{H}) .-{ }^{13} \mathrm{C}$ NMR (DMSO-d ${ }_{6}$ ): $\delta=161.3,148.1,138.0,134.8$, 130.7, 129.7, 128.1, 126.3, 122.9, 120.2, 114.8, 113.2, 105.7. - MS: $\mathrm{m} / \mathrm{z}$ (rel. intensity, \%): $236\left(\left[\mathrm{M}^{+}\right]\right.$, 66), 235 (100), 217 (20).

Representative procedure for the "instant" preparation of coumarins by method $B$

A suspension of $\mathrm{TiCl}_{3}(771 \mathrm{mg}, 5.0 \mathrm{mmol})$, zinc dust ( $653 \mathrm{mg}, 10.0 \mathrm{mmol})$ and substrate 6 a $(630 \mathrm{mg}, 2.51 \mathrm{mmol})$ in THF $(30 \mathrm{ml})$ was refluxed under Ar for $4 \mathrm{~h}$ until TLC showed complete conversion of the starting material. After cooling to room temperature the mixture was filtered through a short pad of silica, the inorganic residue was washed with ethyl acetate $(\approx 50 \mathrm{ml})$ in several portions, the filtrate was evaporated and the residue chromatographed with hexane/ethyl acetate (gradient $4 / 1 \rightarrow 1 / 1$ ) as eluent. Thus, product 12a was obtained as pale-yellow crystals $(510 \mathrm{mg}$, $86 \%$ ).

\section{3,4-Dimethylcoumarin (8i)}

M.p. $110-111^{\circ} \mathrm{C}$ (lit. $112-114^{\circ} \mathrm{C}$ [21]). - IR $\left(\mathrm{cm}^{-1}\right)$ : 2850-3050, 1705, 1625, 1600, 1490, 1460, 1380, 1290, 1150, 1090, 780, 760, 730. - ${ }^{1} \mathrm{H}$ NMR: $\delta=7.20-7.59(\mathrm{~m}, 4 \mathrm{H}), 2.41(\mathrm{~s}, 3 \mathrm{H}), 2.21(\mathrm{~s}$, $3 \mathrm{H}) .{ }^{13} \mathrm{C}$ NMR: $\delta=161.5,151.5,145.5,129.9$, $123.8,123.6,121.8,120.1,116.2,14.5,13.0$. - MS: $\mathrm{m} / \mathrm{z}$ (rel. intensity, \%): $174\left(\left[\mathrm{M}^{+}\right], 100\right), 146(30)$, 131 (46).

\section{4-Methyl-3-phenylcoumarin (8j)}

M.p. $144-146^{\circ} \mathrm{C}$ (lit. $156^{\circ} \mathrm{C}$ [22]). - IR $\left(\mathrm{cm}^{-1}\right)$ : $3060,2940,1715,1620,1610,1490,1450,1380$, 1300, 1180, 1150, 1080, 965, 750, 705. $-{ }^{1} \mathrm{H}$ NMR: $\delta=7.66(\mathrm{dd}, J=1.6,8,1 \mathrm{H}), 7.23-7.56(\mathrm{~m}, 8 \mathrm{H})$, $2.32(\mathrm{~s}, 3 \mathrm{H}) .-{ }^{13} \mathrm{C} \mathrm{NMR}: \delta=160.5,152.3,147.2$, $134.0,130.9,129.6,128.0,127.9,127.8,124.7,123.8$, 120.1, 116.4, 16.1. - MS: $\mathrm{m} / z$ (rel. intensity, \%): $236\left(\left[\mathrm{M}^{+}\right], 100\right), 235$ (65), 208 (37), 207 (49), 178 (21), 131 (20), 89 (14), 77 (8).

\section{4-Amino-3-phenylcoumarin (12a)}

M.p. $179-180^{\circ} \mathrm{C}$ (lit. $182^{\circ} \mathrm{C}$ [23]). - IR $\left(\mathrm{cm}^{-1}\right)$ : $3430,3340,3250,1735,1625,1610,1590,1575$, 1525, 1440, 1290, 1250, 1220, 755, 705. - ${ }^{1} \mathrm{H}$ NMR $\left(\mathrm{DMSO}^{\left.-\mathrm{d}_{6}\right):} \delta=8.15(\mathrm{dd}, J=8.6,1.7,1 \mathrm{H})\right.$, $7.25-7.65$ (m, 8H), 6.75 (br s, $2 \mathrm{H}$ ). $-{ }^{13} \mathrm{C}$ NMR $\left(\right.$ DMSO-d $\left._{6}\right): \delta=160.7,152.4,150.6,133.6,131.7$, $130.8,128.4,127.0,123.4,123.2,116.3,114.4$, 97.4. - MS: $m / z$ (rel. intensity, \%): $237\left(\left[\mathrm{M}^{+}\right], 100\right)$, 236 (61), 209 (26), 180 (28), 165 (10), 120 (16), 90 (14), 77 (11).

\section{Acknowledgement}

Financial support by the Volkswagen-Stiftung, Hannover, is gratefully acknowledged. 
[1] a) J. E. McMurry, Chem. Rev. 89, 1513 (1989);

b) D. Lenoir, Synthesis 1989, 883;

c) C. Betschart, D. Seebach, Chimia 43, 39 (1989);

d) A. Fürstner, Angew. Chem. 105, 171 (1993); Angew. Chem., Int. Ed. Engl. 105, 164 (1993).

[2] a) A. Fürstner, D. N. Jumbam, Tetrahedron 48, 5991 (1992);

b) A. Fürstner, D. N. Jumbam, H. Weidmann, Tetrahedron Lett. 32, 6695 (1991);

c) A. Fürstner, D. N. Jumbam, G. Seidel, Chem. Ber. 127, 1125 (1994);

d) Pyrroles: A. Hupperts, A. Fürstner, unpublished; e) A. Fürstner, A. Ernst, Tetrahedron 51, 773 (1995).

[3] A. Fürstner, D. N. Jumbam, J. Chem. Soc. Chem. Commun. 1993, 211.

[4] A. Fürstner, A. Hupperts, A. Ptock, E. Janssen, J. Org. Chem. 59, 5215 (1994).

[5] J. E. McMurry, M. G. Silvestri, M. P. Fleming, T. Hoz, M. W. Grayston, J. Org. Chem. 43, 3249 (1978).

[6] C. Floriani, G. Fachinetti, J. Chem. Soc. Chem. Commun. 1972, 790.

[7] a) R. Schobert, Angew. Chem. 100, 869 (1988); Angew. Chem., Int. Ed. Engl. 27, 855 (1987);

b) S. Dürr, U. Höhlein, R. Schobert, J. Organomet. Chem. 458, 89 (1993).

[8] a) T. Mukaiyama, H. Sugimura, T. Ohno, S. Kobayashi, Chem. Lett. 1989, 1401;

b) H. Takeshita, A. Mori, S. Nakamura, Bull. Chem. Soc. Jpn. 57, 3152 (1984);

c) N. Kato, H. Takeshita, Bull. Chem. Soc. Jpn. 58, 1574 (1985).

[9] a) $\alpha$-Ketoacid chlorides: H. C. J. Ottenheijm, J. H. M. De Man, Synthesis 1975, 163;

b) for a convenient preparation of $\alpha$-ketoacids see: J. S. Nimitz, H. S. Mosher, J. Org. Chem. 46, 211 (1981).

[10] Schobert et al. and Geise et al. reported similar deoxygenation reactions occurring with vic-diketones. The precise mechanism for this Clemmensen-type reduction has not yet been established, $c f$. ref. [7a] and: Y. Dang, H. J. Geise, Bull. Soc. Chim. Belg. 100, 375 (1991).
[11] a) A. Fürstner, H. Weidmann, Synthesis 1987, 1071; b) A. Fürstner, R. Csuk, C. Rohrer, H. Weidmann, J. Chem. Soc. Perkin Trans. 1, 1729 (1988);

c) D. L. J. Clive, C. Zhang, K. S. K. Murthy, W. D. Hayward, S. Daigneault, J. Org. Chem. 56, 6447 (1991).

[12] H. Bönnemann, B. Korall, Angew. Chem. 104, 1506 (1992); Angew. Chem., Int. Ed. Engl. 31, 1490 (1992). We thank Prof. Bönnemann and his coworkers for providing a sample of the titanium clusters.

[13] a) A. Clerici, O. Porta, Synthesis 1993, 99; b) A. Clerici, O. Porta, J. Org. Chem. 47, 2852 (1982).

[14] J. E. McMurry, T. Lectka, J. G. Rico, J. Org. Chem. 54, 3748 (1989).

[15] Only recently two in-depth investigations on the actual nature of the McMurry agents prepared by reduction of $\mathrm{TiCl}_{3}$ with $\mathrm{Mg}$ or $\mathrm{LiAlH}_{4}$, respectively, have been reported $c f$. a) L. E. Aleandri, S. Becke, B. Bogdanovic, D. J. Jones, J. Roziere, J. Organomet. Chem. 472, 97 (1994);

b) L. E. Aleandri, B. Bogdanovic, A. Gaidies, D. J. Jones, S. Liao, A. Michalowicz, J. Roziere, A. Schott, J. Organomet. Chem. 459, 87 (1993).

[16] L. Capuano, A. Ahlhelm, H. Hartmann, Chem. Ber. 119, 2069 (1986).

[17] S. D. Work, D. R. Bryant, C. R. Hauser, J. Org. Chem. 29, 722 (1964).

[18] F. Künzle, J. Schmutz, Helv. Chim. Acta 53, 798 (1970).

[19] W. Stadlbauer, T. Kappe, Synthesis 1981, 833.

[20] W. Stadlbauer, O. Schmut, T. Kappe, Monatsh. Chem. 111, 1005 (1980).

[21] J. Motoyoshiya, A. Teranishi, R. Mikoshiba, I. Yamamoto, H. Gotoh, J. Enda, Y. Ohshiro, T. Agawa, J. Org. Chem. 45, 5385 (1980).

[22] P. Pulla Rao, G. Srimannarayana, Synthesis 1981, 887.

[23] W. Stadlbauer, T. Kappe, Monatsh. Chem. 115, 467 (1984). 